\title{
Hybrid Magnet - Field Winding Solutions for Exciters of Synchronous Generators
}

\author{
Giovanni Decuzzi \\ Power Electronics,Machines and \\ Control Group \\ University of Nottingham,UK \\ Paolo Giangrande \\ Power Electronics,Machines and \\ Control Group \\ University of Nottingham,UK
}

\author{
Stefano Nuzzo \\ ${ }^{1}$ Power Electronics,Machines and \\ Control Group \\ University of Nottingham,UK \\ Michael Galea \\ Power Electronics,Machines and \\ Control Group \\ University of Nottingham,UK
}

\author{
Paolo Bolognesi \\ Electrical Machines, Power Electronics \\ and Drives Group \\ Universityof Pisa,Italy \\ p.bolognesi@ieee.com
}

\begin{abstract}
For power generation purposes, wound-field synchronous generators are often preferred to permanent magnet machines, due to the need of controlling the field winding voltage and current for guaranteeing a constant voltage at the generator's armature terminals. In a traditional generating set, this function is accomplished by a relatively complex feedback control system which typically comprises an excitation system and an automatic voltage regulator. Due to their excellent and proven performance capability, such classical generating sets have seen only strictly incremental improvements in the last 60 years or so. However, today, there is an interest in revamping their design and development, partly due to the even increasing efficiency and reliability requirements and partly due to advances in material and manufacturing techniques. This work investigates the feasibility of a hybrid permanent magnet - field winding excitation for synchronous generators' exciters. This design solution aims at reducing the losses of the exciter and thus of the whole generating system, while also increasing its reliability.
\end{abstract}

Keywords-Brushless exciter, Hybrid excitation, Excitation systems, Permanent magnets, Synchronous generators.

\section{INTRODUCTION}

In several applications ranging from power plants to aircraft, the power generation historically relies on the employment of the wound-field synchronous generator (SG) [1]. Through the years, this consolidated and durable technology has proven its effectiveness by revealing high reliability and efficiency. Besides these benefits, the capability of easily control the voltage at its armature terminals represents the advantage that mainly concurred to the wound-field SG success. The voltage regulation task is fulfilled by a relatively complex feedback control system, whose core is represented by the excitation system. Standard definitions and guides for the preparation of excitation systems are provided in [2] and [3].

The aspects relative to the field current control and the armature voltage regulation have been matter of study since 1900 [4]. Before the advent of the semiconductor devices, the excitation systems were largely based on the DC commutator machine (DC commutation systems) which was not only used as a main and pilot exciter, but also as a control amplifier. This solution requires brushes and slip rings for feeding the SG field and it is generally characterized by a slow response. To overcome these challenges, both brushless [5] and static
[6] excitation systems are widely employed nowadays. In small-to-medium power generating sets (gensets), the most typical configuration is the brushless AC excitation system consisting of a three-phase "inside-out" generator (i.e. the exciter with field and armature windings respectively located on the stator and rotor) and a six-pulse rotating diode rectifier $[7,8]$. The brushless system is implemented either in the selfexcited form, where the voltage build-up on initial run-up relies on the residual magnetism of the $S G$, or in the separately-excited layout, where a permanent magnet generator (PMG) is added to the AC brushless excitation system and used as a pre-exciter [9]. The latter architecture presents a better voltage build-up capability on initial run-up and a higher protection of the automatic voltage regulator (AVR) from severe transient fault conditions. In some cases, the field winding of the exciter is fully replaced by a PM excitation. However, this configuration implicates the installation of a controlled rectifier (e.g. a thyristor bridge) to fulfil the field current control objective [10]. A number of variants have been proposed in the recent years, including dual three phase excitation systems [10], brushless layout with dc-to-dc converter [11] and harmonic excitation systems [12].

In this work, an alternative and simpler configuration is presented. The concept consists in supporting the field winding of the existing exciter with an additional excitation provided by PMs, thus resulting in a hybrid excitation architecture [13] aimed at increasing both the reliability and efficiency of the entire genset. In order to demonstrate the effectiveness of such hybrid excitation solution, first an offthe-shelf self-excited genset is considered as benchmark case study and its exciter is investigated. This relatively small electric machine is described and analyzed in detail via a purposely-built finite-element (FE) model. For the genset under study, the optimal hybrid exciter (field winding + PMs) is identified through a trade-off study and its design is accomplished. Finally, the performance of the designed hybrid structure is compared against that of the original exciter for several SG's loading conditions. The outcome of the comparative analysis allowed to confirm the perceived advantages and the possible drawbacks of the presented hybrid excitation approach. 


\section{FE ANALYSIS OF THE BENCHMARK EXCITER}

As mentioned above, this section is dedicated to the description of the exciter chosen as benchmark and the FE model used to analyze its electromagnetic performance is also discussed.

\section{A. Description of the considered excitation system}

The genset under investigation is a self-excited 400kVA, $400 \mathrm{~V}$ at $50 \mathrm{~Hz}$ system, composed by

1) the main alternator, i.e. a 4 poles, three-phase salient-pole SG;

2) the brushless $\mathrm{AC}$ excitation system, i.e. an "inside-out" machine whose armature terminals feed a six-pulse rotating diode bridge;

3) the AVR, whose function is to ensure that the output voltage of the main SG is kept constant at its rated value.

A schematic of such genset layout is sketched in Fig. 1.

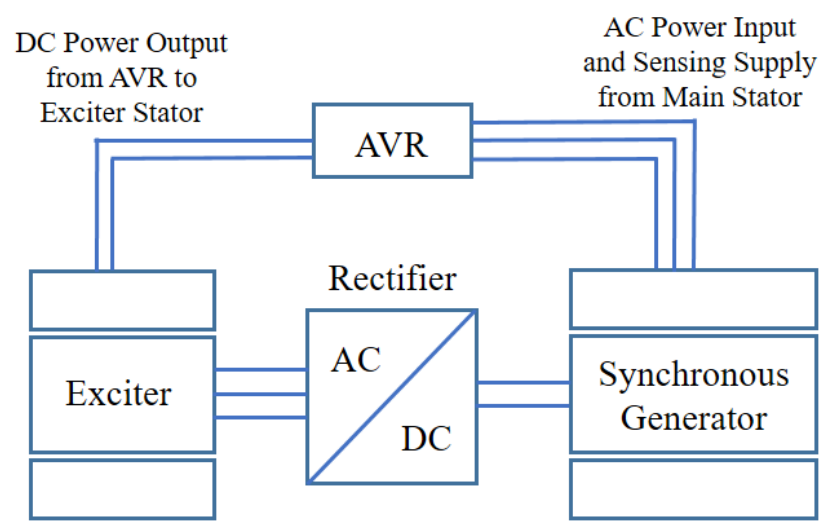

Fig. 1. A qualitative schematic of the self-excited power genset under study.

It is noteworthy to point out that the work bestowed in the present publication focuses on the only the exciter. Indeed, the aspects related to the modeling, analysis and design of the main alternator were widely discussed in [14-16], whilst the challenges associated with the diode commutations occurring in the diode bridge can be found in [7], [17] and [18].

In the excitation system, the exciter operates as a generator. Contrarily to the main alternator, the field winding is wound on the stationary ferromagnetic structure of the machine, while its armature winding is placed inside the rotor slots. The examined exciter is a 14 poles machine, designed to provide $50 \mathrm{~A}$ and $100 \mathrm{~V}$ to the main SG field winding when the latter operates at full-load. The rotor structure consists of 42 slots which accommodate a star-connected, three-phase winding. A single-layer, full-pitched winding layout is employed, meaning that there is 1 slot-per-pole-per-phase. The material used for both stator and rotor is a standard M700-65A. To take advantage of the space available along the radial direction inside the frame enclosing the whole genset, the exciter presents the same outer diameter as the main alternator. This choice leads to a quite compact design for the exciter due to the high ratio between outer diameter and axial length (i.e. 10 in the specific platform under study).

\section{B. FE Model of the benchmark exciter}

Having described the benchmark genset and the major design features of the exciter, its electromagnetic performance is evaluated via a FE analysis. As previously mentioned, the exciter is characterized by a high outer diameter / axial length ratio and this indicates that the end windings can play an important role on the machine operations, from both the resistive and electromagnetic points of view. Therefore, a 3-D analysis would be the most suitable approach for this type of electric machine, however coming at the cost of increasing the computation time of the FE solver. Nevertheless, the FE analysis is used as comparative tool in this study. In fact, the benchmark exciter performance is going to be compared against the one of the proposed hybrid excitation design. Since the only difference between the two machines stands in the presence of the PMs, it is reasonable to assume that the two exciters present the same 3-D features. Thus, both sets of 3-D FE results would be affected by equal "offsets" (i.e. the 3-D behavior), which would be cancelled out during the performance comparison. It is for this reason that a 2-D approximation of the electromagnetic problem can be employed without compromising the investigation validity. Hence, a 2-D FE model of the exciter is built. To further reduce the computation burden, the machine symmetry is exploited by modelling only $1 / 14^{\text {th }}$ of the whole exciter (i.e. angular sector corresponding to one pole pitch), as shown in Fig. 2.

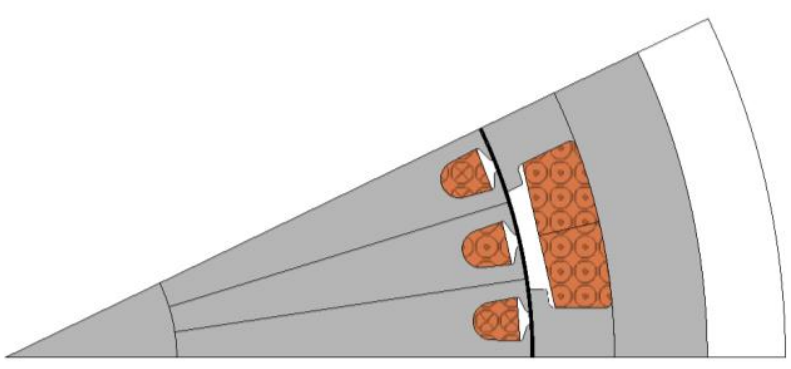

Fig. 2. 2-D FE model of the benchmark exciter (1/14 $4^{\text {th }}$ of the entire machine).

The described FE model is thus used to evaluate the no-load and the on-load (with the main SG armature winding opencircuited) behavior of this small "inside out" machine.

\section{No-load operation of the benchmark exciter.}

The first FE test carried out on the benchmark exciter consists in evaluating its no-load operation. The simulation is performed with the rotor windings terminals open circuited and stator field winding fed with a DC current equal to 0.9A. The current value of $0.9 \mathrm{~A}$ represents the current flowing through the exciter field winding when the main SG operates at rated speed and voltage at no-load condition. In Fig.2, the no-load phase voltages induced in the three rotor phases are shown. It can be observed the trapezoidal trend of the waveforms, due to the simple structure of the armature winding (single-layer, one slot-per-pole-per-phase) and to the geometry of the stator poles. However, a trapezoidal trend of the output voltage waveforms is desired for the application at hand, given that the diode rectifier is interposed between exciter and main SG.

\section{Rated no-load operation of the main alternator}

To operate the main generator at rated no-load condition, i.e. at rated speed and armature voltage, the exciter armature winding terminals are connected to the diode rectifier, which in turn supplied the main SG field winding. In such conditions, the exciters is obviously loaded. For both main SG and exciter, the described operating conditions are very 


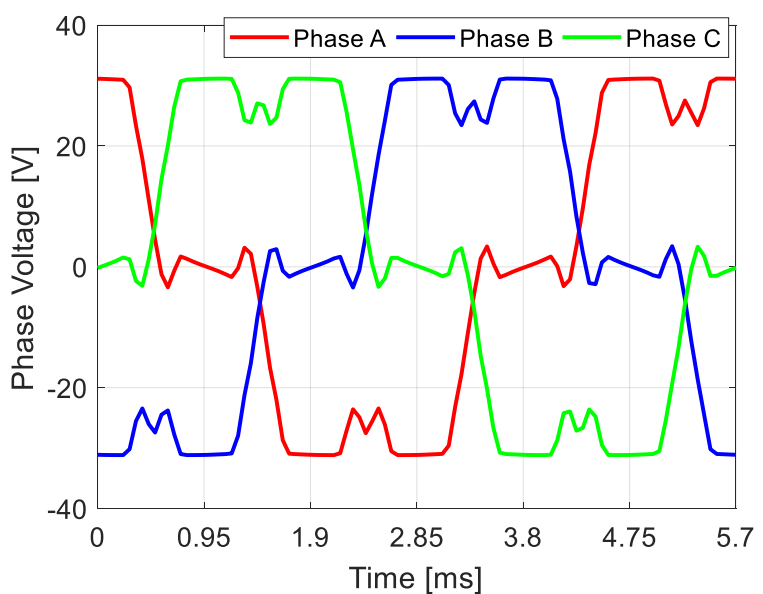

Fig. 3. No-load phase voltage waveforms induced in the rotor armature windings, when the field current is equal to $0.9 \mathrm{~A}$.

important for the sake of this study. In fact, as explained later in the paper, the selected operating point corresponds to the chosen objective for the design of the proposed hybrid excitation concept. In other words, the SG no-load rated operation must be ensured by only the PMs contribution in the hybrid architecture. To simulate such working point, the FE model is coupled to the electric circuit shown in Fig. 4, where it can be noticed that the SG field winding is represented through an R-L load. Due to its nature, the main SG field winding features a significant inductance value, which leads to a considerable time constant. In terms of FE evaluations, this would result in a long simulation time as the time window needs to be set long enough for reaching the steady-state. In order to contain the computation time of the FE solver, a purely resistive load is used and the average values of the rectified voltage and current flowing through the SG rotor winding are considered.

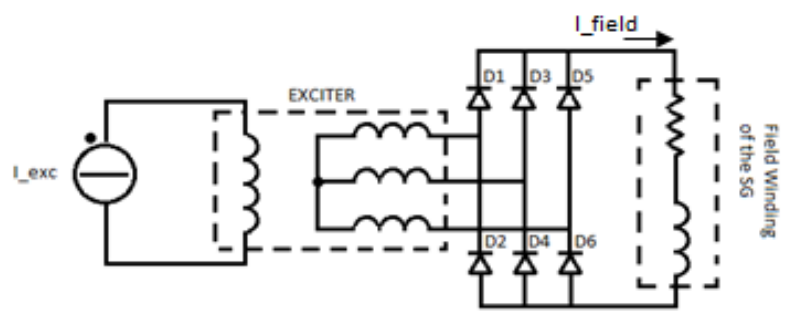

Fig. 4. Electric circuit coupled to the FE model of the exciter.

A transient with motion simulation has been run to accurately evaluate these quantities. The exciter field current (I_exc in Fig. 4) is set at 0.9A (as indicated in Section II.C). The AC exciter phase voltages are depicted in Fig. 5, where the distortion due to the presence of the diode bridge can be noticed. In Fig. 6, the current flowing inside the main generator field winding is reported. In this figure, also the average value of such current is highlighted. It can be seen that this is equal to $\approx 14.3 \mathrm{~A}$, which is just the value needed by the main SG to provide the rated voltage at its armature terminals when these are open circuited.

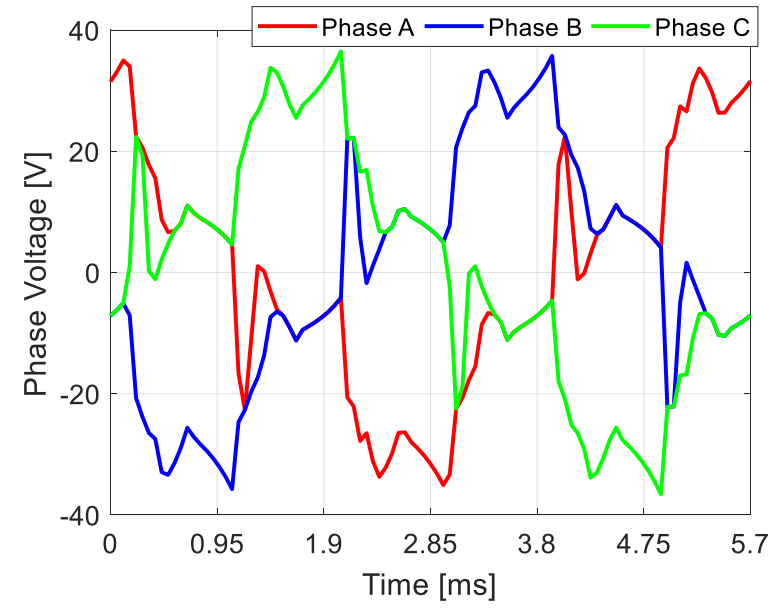

Fig. 5. Phase voltages induced in the exciter rotor windings at rated no-load operation of the main SG.

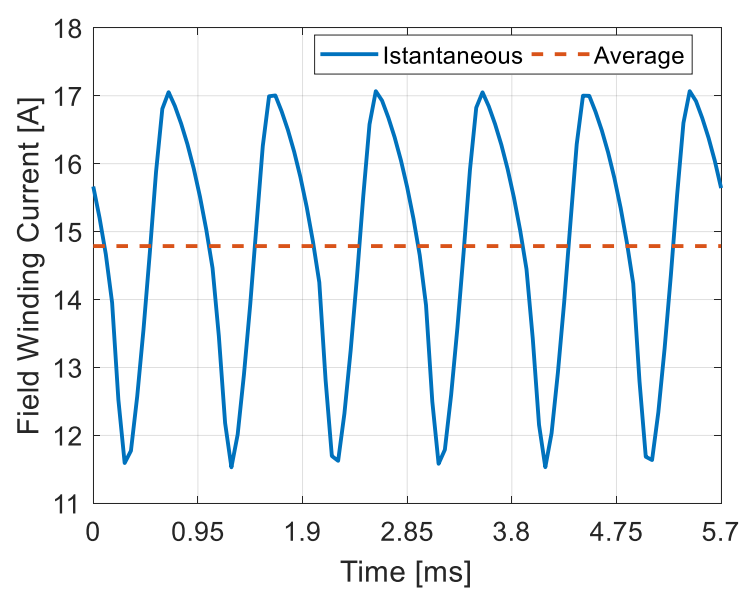

Fig. 6. Current flowing through the field circuit of the main SG during its rated no-load operation: istantaneous (blue) and average (red) current values.

Although additional working conditions of the main alternator are worthy to be analyzed (e.g. its rated full-load), the two operations described in Section II.C and II.D are sufficient for designing the hybrid excitation. Indeed, this proposed hybrid solution aims to fulfil the field requirements of the main SG during its rated no-load operation.

\section{PROPOSED CONCEPT: THE HYBRID EXCITATION}

Before going into the design details, it is noteworthy recalling same primary aspects regarding the proposed hybrid excitation solution. Firstly, the hybrid excitation aims to provide the "basic contribution" to the SG field winding via the PMs. In other words, the hybrid exciter is sized selecting as operating point the field winding current and voltage values of the main $\mathrm{SG}$, such that the latter develops the rated voltage of $400 \mathrm{~V}$ at no-load condition. Secondly, the "basic contribution" is chosen upon the philosophy of keeping unchanged the electronic circuit (embedded into the AVR) which feeds the exciter field winding. Since the chosen genset is designed for isolated operation and for supplying ohmic-inductive loads, it is then clear that the selected operating point permits the use of only positive values of the exciter field current. This feature allows to keep unmodified the existing unidirectional power electronics. 


\section{A. Benefits and drawbacks}

Besides the well-known advantages that the PMs present against excitation windings, the main benefits resulting from the implementation of the hybrid excitation onto the considered applications can be summarized as follows:

1) Reduced exciter field current and losses in any operating condition of the main alternator - while obviously at noload no excitation current would be required due to the PMs presence, at load operation a significant reduction of the exciter field current is expected with an optimal design of the hybrid solution.

2) Improved lifetime of the exciter- the field losses reduction would lower the operating temperatures, thus easing the thermal management [19], reducing the thermal aging and consequently enhancing the machine lifetime.

3) Improved exciter design - as opposed to Point 2), the hybrid exciter could be operated at the same rating as the original machine, thus allowing for a reduction of the copper volume for the same current. This can enable the exciter redesign [18].

4) Long-term economic impact - since losses are wasted energy and are thus associated to direct and indirect costs, the field loss reduction would provide appreciable savings over the entire lifecycle of the genset.

5) Higher reliability - the presence of PMs would ensure a continuous operation of the genset even in the case of a fault occurring in the exciter field winding, providing a voltage level equal to its rated value. In addition, the PMs would permit a more reliable voltage build-up during start-up compared to the benchmark, self-excited system, where this relies upon the materials' residual magnetism.

The hybrid excitation concept drawbacks include

1) possibly higher production costs inherently deriving from the use of PMs;

2) potential issues deriving from a fault occurring in the exciter armature winding, in the diode rectifier or even in the generator;

3) impossibility of controlling the voltage build-up from zero terminal voltage.

\section{B. Selection of the design configuration}

Having described the proposed concept and its major perceived advantages when implemented onto the considered application, the design of the hybrid exciter can begin. As a first step, the most appropriate PMs location needs to be identified considering the available geometry of the benchmark exciter. This choice is mainly driven by the necessity of minimizing the alterations of the existing exciter geometry (i.e. major changes of the exciter stator core are preferably avoided in the current investigation). In this perspective, it is clear that the "quasi-zero" modification would consist in placing the PMs directly in the exciter airgap. Such solution would be feasible for the benchmark exciter, due to its airgap thickness (i.e. $1 \mathrm{~mm}$ ). Supplementary considerations regarding the PMs characteristics are listed below:

1) the PMs must be inserted in such a way that they do not distort and/or interfere with the field map created by the excitation windings;
2) the PMs should be magnetized in such a way as to strengthen the field map;

3) the PMs should not be source of excessive magnetization in the ferromagnetic core, thus preventing its magnetic saturation.

Accounting the above considerations, two possible ways of realization of the hybrid machine are possible: 1) the surfacemounted [20] and 2) surface-inset [21] layouts. The latter may lead to undesirable effects in correspondence of the magnet edges and it would come at the cost of realizing a slot on the main surface of each stator tooth. Another important aspect is that the airgap thickness under the main stator pole would be non-uniform, potentially resulting in a high harmonic content of the output voltage. Considering all the above, a surface-mounted solution is adopted as this would imply a minimal design modification consisting in removing a very small layer of tooth head. In Fig. 7, the surfacemounted PM can be observed in blue.

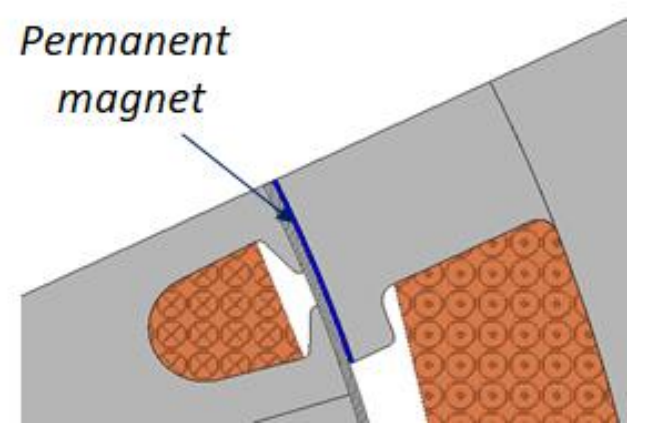

Fig. 7. The proposed hybrid magnet - field winding solution for the exciter.

\section{Selection of PMs}

Once the PMs location has been identified, the choice of the PMs material represent the second step of the design process. The PMs material is chosen according to the following requirements:

1) Relatively high coercive field $\left(\mathrm{H}_{\mathrm{c}}\right)$ and remanence flux density $\left(\mathrm{B}_{\mathrm{r}}\right)$ are needed to minimise the PMs thickness. In fact, the resulting equivalent airgap increase (when a surface-mounted configuration is used) may potentially lead to higher exciter field currents during load operation compared to the one demanded in absence of PMs (i.e. benchmark exciter).

2) Relatively high stability with the temperatures is advisable, as the exciter operating temperature is assumed to be the same as the main generator, i.e. $150^{\circ} \mathrm{C}$.

Taking into account these requirements, Samarium Cobalt magnets are selected to achieve the objectives of this work. The main PM features are summarized in Table I, where the achievements in terms of small thickness (relatively to the main mechanical airgap thickness, i.e. $1 \mathrm{~mm}$ ) and good performance at high temperatures (more specifically at the considered $150^{\circ} \mathrm{C}$ ) are provided. In particular, the PMs thickness is a result of an accurate trade-off study aimed at achieving the rated no-load requirements of the main SG and at minimizing the equivalent airgap. The latter thus becomes equal to $1.5 \mathrm{~mm}$ (in correspondence of the stator teeth) in the proposed hybrid excitation design: $1 \mathrm{~mm}$ of mechanical airgap +0.5 of PMs. To achieve this objective, all the space available along the tangential development of the stator teeth 
is used to allocate the PMs. The angular span of the magnets therefore represents a design constraint in the trade-off investigation and this concept is observable in Fig. 7.

TABLE I. PROPERTIES OF THE SELECTED PMs

\begin{tabular}{|l|c|}
\hline \multicolumn{2}{|c|}{ SmCo_18/30 } \\
\hline Operating temperature $\left[{ }^{\circ} \mathrm{C}\right]$ & 150 \\
\hline PMs thickness $[\mathrm{mm}]$ & 0.5 \\
\hline Coercive field at $150^{\circ} \mathrm{C}[\mathrm{A} / \mathrm{m}]$ & 619925 \\
\hline Magnetic permeability at $150^{\circ} \mathrm{C}$ & 1.06 \\
\hline
\end{tabular}

\section{FE RESUlts AND COMPARISON AGAINST BENCHMARK EXCITER}

In this section, the performance comparison between the hybrid exciter (i.e. PM + field winding) and the conventional exciter (i.e. only field winding) is presented. The comparative exercise aims to demonstrate that a reduction of the excitation current is achievable by adopting the proposed solution. Although in the first place this outcome might seem expected, obtaining an appreciable field current reduction is anything but trivial when the main SG operates at load condition. In fact the PMs insertion provides a null-current flux, however a $50 \%$ increase of the equivalent airgap thickness (from $1 \mathrm{~mm}$ to $1.5 \mathrm{~mm}$ ) is required for their placement. The perceived improvement resulting from the implementation of the hybrid exciter is verified by carrying out a FE comparative analysis. In Fig. 8, the comparison between the no-load voltage waveforms relative to one machine armature phase is illustrated. In this test, while for the benchmark exciter a field current of 0.9A has been used in the simulation, a null current has been instead set for the field winding of the hybrid solution. From Fig. 8, it can be observed that the hybrid exciter ensures the same no-load performance as the original machine fulfilling the design target.

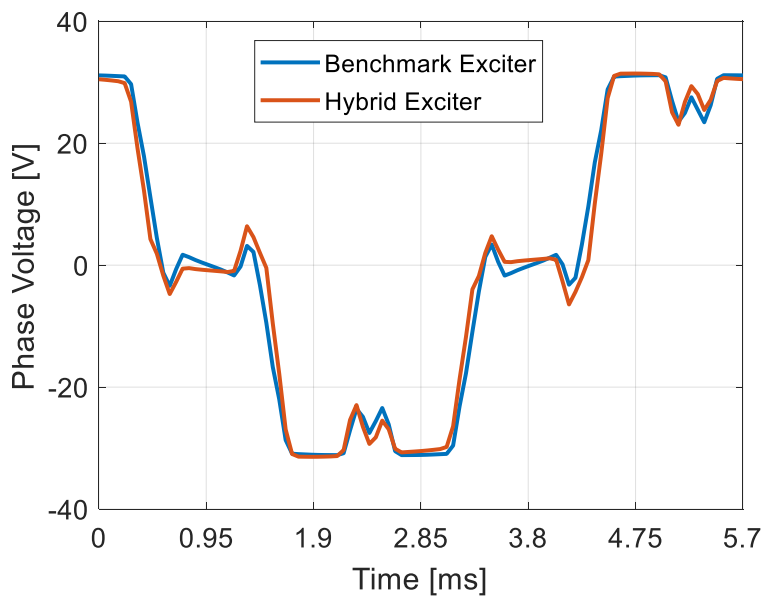

Fig. 8. Comparison between the no-load phase voltage waveforms induced in the benchmark (with 0.9A flowing in its field winding) and the hybrid exciters (with null excitation current).

Established the validity of the hybrid excitation concept at no-load operation, the behavior of both exciters is evaluated at on-load operation, i.e. the exciter armature circuit connected to the main SG field winding via the diode rectifier. The comparison outcome is depicted in Fig. 9, where a slight increase of the current ripple can be noticed when the hybrid excitation is employed. Further, by adopting the proposed solution, a modest decrease is seen in the average field current of the main SG.

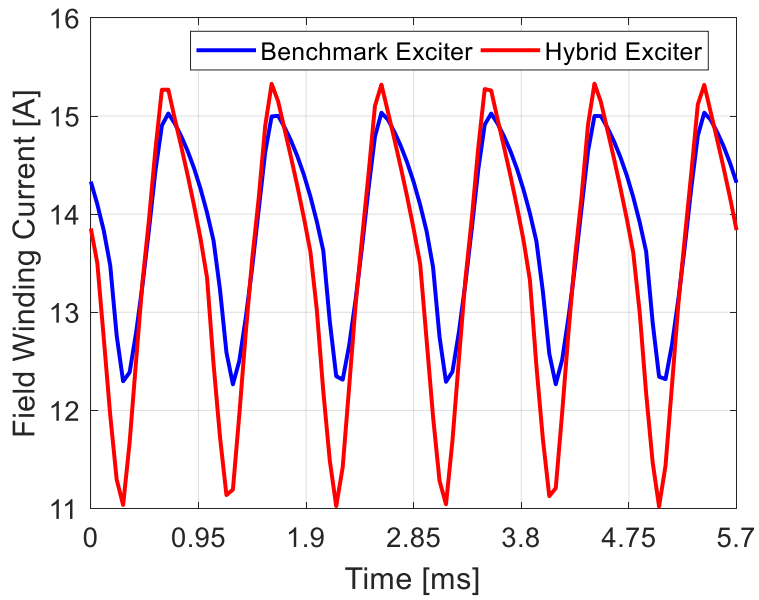

Fig. 9. Main SG field currents at no-load rated condition provided by both hybrid (blue) and benchmark (red) exciters.

Having thus confirmed that the hybrid excitation design achieves very similar performance as the original machine at no-load operation of the exciter (see Fig. 8) and of the main generator (see Fig. 9), the final step of the comparative study consists in assessing the operating capability of the hybrid exciter at several loading conditions of the main SG. In particular, three different operating points of the main SG, namely $50 \%, 100 \%$ and $110 \%$ of the full-load, are investigated. The obtained results are listed in Table II. Considering the full-load operation, a $25.5 \%$ exciter field current (I_exc) reduction is reached when the hybrid design is employed, while the mismatch in terms of SG field current (I_field) is negligible. The comparison outcomes respectively at the $50 \%$ and at the $110 \%$ of the full-load of the main SG similarly highlight a significant decrease of the exciter field current $(51.6 \%$ and $22 \%$ respectively). The findings presented throughout this section allow to conclude that the hybrid exciter (PMs + field winding) represents a very interesting solution for small-to-medium power gensets, as compared to those implementing conventional exciters (only field winding). In fact, the significant loss reduction achieved could result in the operational and economic benefits highlighted in Section III.A. In addition to these, a more reliable system is obtained through the use of the hybrid excitation.

TABLE II. COMPARISON BETWEEN BENCHMARK AND HYBRID EXCITERS AT SEVERAL OPERATING CONDITIONS OF THE MAIN SG.

\begin{tabular}{|c|c|c|c|c|}
\hline $\begin{array}{c}\text { Loading } \\
\text { Condition }\end{array}$ & $\begin{array}{c}\text { Exciter and SG } \\
\text { field current }\end{array}$ & $\begin{array}{c}\text { Benchmark } \\
\text { Exciter }\end{array}$ & $\begin{array}{c}\text { Hybrid } \\
\text { Exciter }\end{array}$ & Difference \\
\hline \multirow{2}{*}{$100 \%$} & I_Exc [A] & 2.35 & 1.75 & $-25.5 \%$ \\
\cline { 2 - 5 } & I_Field [A] & 50.6 & 50.28 & $-0.63 \%$ \\
\hline \multirow{2}{*}{$50 \%$} & I_Exc [A] & 1.55 & 0.75 & $-51.6 \%$ \\
\cline { 2 - 5 } & I_Field [A] & 30.4 & 30.01 & $-0.98 \%$ \\
\hline \multirow{2}{*}{$110 \%$} & I_Exc [A] & 2.50 & 1.95 & $-22.0 \%$ \\
\cline { 2 - 5 } & I_Field [A] & 54.4 & 53.3 & $-1.96 \%$ \\
\hline
\end{tabular}




\section{CONCLUSIONS}

The main objective of this paper was to investigate the feasibility of designing a hybrid exciter for synchronous generators, where permanent magnets are added to support the existing field winding in producing the required excitation flux. This work was motivated by the aim of improving both performance and reliability of the overall generating system.

As vehicle to study the proposed concept, a $400 \mathrm{kVA}$ power genset equipped with a brushless excitation system was considered and special focus was given to the exciter. First, the benchmark exciter was analyzed via a purposelybuilt FE model. Then, the hybrid excitation concept was investigated with special attention given to the PM location and to the design layout. Taking into account several operating conditions, a comparison between the FE results of the existing and the proposed designs concluded the work.

Considering the rated no-load operation of the main SG, the hybrid excitation solution did not require field current for feeding the main SG field winding, since the PMs were designed to provide the necessary excitation at this specific working point. Evaluating the on-load operation of the main SG, the study showed a reduction of $51.6 \%, 25.5 \%$ and $22 \%$ of the excitation current respectively at $50 \%, 100 \%$ and $110 \%$ of the rated load of the main generator. Therefore, it can be concluded that the hybrid solution can potentially lead to significant benefits to the whole genset, including

1) Reduced exciter losses - although this would not significantly enhance the overall generating set efficiency in absolute terms (in fact, typically, such apparatus already feature high efficiencies), the operational (i.e. better thermal management, improved lifetime, etc.) and the economic (i.e. cumulated savings over the system lifetime, e.g. reduced fuel consumption) benefits can be significant;

2) Increased reliability of the whole system ensured by

a) a stable and safe voltage build-up during the startup phase, with no need of an extra permanent magnet pilot exciter;

b) the fault-tolerance introduced by the permanent magnets with respect to any fault occurring in the field winding of the exciter .

Finally, the achieved field current reduction provides room for decreasing the exciter weight and size by optimizing the wire cross-section and the stator slots size. Hence, it would be possible shortening the stator poles and thus shrinking the external diameter of the machine. All these aspects will be examined in future works.

\section{REFERENCES}

[1] V. Madonna, P. Giangrande, and M. Galea, "Electrical Power Generation in Aircraft: review, challenges and opportunities," in press on IEEE Transactions on Transportation Electrification, 2018, DOI: 10.1109/TTE.2018.2834142.

[2] IEEE Standard Definitions for Excitation Systems for Synchronous Machines, IEEE Std. 421.1-2007 (Revision of IEEE Std. 421.1-1986), pp. 1-33, 2007.

[3] IEEE Guide for the Preparation of Excitation System Specifications, IEEE Std. 421.4, 2014.

[4] D.R. Fenwick, C.Eng., F.I.E.E. and W.F. Wright, B.Sc, C.Eng., M.I.E.E., "Review of trends in excitation systems and possible future developments", Proceeding of the Istitution of Electrical Engineers, vol. 123, May 1976.

[5] T.L. Dillman, F.W. Keay, C. Raczkowsi, J.W. Skooglund and W.H. South, "Brushless excitation" IEEE Spectr., vol. 9, no. 3, pp. 58-66, Mar. 1972.

[6] R.C. Schaefer, "Applying Static Excitation Systems", IEEE Ind. App. Society Mag., vol. 4, no 6, Nov.1998.

[7] S. Nuzzo, M. Galea, C. Gerada and N. Brown, "Analysis, Modeling, and Design Considerations for the Excitation Systems of Synchronous Generators," in IEEE Transactions on Industrial Electronics, vol. 65, no. 4, pp. 2996-3007, April 2018.

[8] V. Ruuskanen, M. Niemela, J.Pyrhonen, S.Kanerva, J.Kaukonen, "Modelling the Brushless excitation system for Synchronous Machine", IET Electronic Power Applications, 2008.

[9] G. Laliberte, “A comparison of generator excitation systems (Power topic 6008 - Technical information from Cummins Power Generation)," in Technical Report GLPT-6008-EN (10/14), Oct 2014, pp. 1-6.

[10] J. K. Nøland, F. Evestedt, J. J. P'erez-Loya, J. Abrahamsson, and U. Lundin, "Comparison of thyristor rectifier configurations for a sixphase rotating brushless outer pole PM exciter," IEEE Trans. on Ind. Electron., vol. 65, no. 2, pp. 968-976, Feb 2018.

[11] J. K. Nøland, F. Evestedt, J. J. P'erez-Loya, J. Abrahamsson, and U. Lundin, "Testing of active rectification topologies on a sixphase rotating brushless outer pole pm exciter," IEEE Trans. Energy Convers., vol. 33, no. 1, pp. 59-67, March 2018.

[12] F. Yao, Q. An, L. Sun, and T. A. Lipo, "Performance investigation of a brushless synchronous machine with additional harmonic field windings," IEEE Trans. Ind. Electron., vol. 63, no. 11, pp. 6756-6766, Nov 2016.

[13] P. Bolognesi, F. Papini, O. Bruno, "Analysis of a Fault-Tolerant Hybrid-Excitation DC Motor," Compel, vol. 29, n. 5 November 2010, pp. 1361-1379.

[14] S. Nuzzo, P. Bolognesi, G. Vakil, D. Fallows, C. Gerada, N. L. Brown, M. Galea, "A Methodology to Remove Stator Skew in Small-Medium Size Synchronous Generators via Innovative Damper Cage Designs," in IEEE Transactions on Industrial Electronics, vol. 66, no. 6, pp. 42964307, June 2019.

[15] Y. Wang, G. Vakil, S. Nuzzo, M. Degano, M. Galea, C. Gerada, H. Zhang, N. Brown, "Sensitivity analysis for performance and power density improvements in salient-pole synchronous generators," 2017 IEEE Workshop on Electrical Machines Design, Control and Diagnosis (WEMDCD), Nottingham, 2017, pp. 163-168.

[16] C. Spagnolo, S. Nuzzo, G. Serra, C. Gerada and M. Galea, "Analysis of salient-pole synchronous generators operating in single-phase condition," 2017 IEEE Workshop on Electrical Machines Design, Control and Diagnosis (WEMDCD), Nottingham, 2017, pp. 33-38.

[17] S. Nuzzo, M. Galea, C. Gerada, N. Brown, "Prediction of the voltage drop due to the diode commutation process in the excitation system of salient-pole synchronous generators", Proc. in the $19^{\text {th }}$ International of Electrical Machines and Systems, (ICEMS 2016), 2016.

[18] S. Nuzzo, M. Galea, C. Gerada and N. L. Brown, "An analytical and genetic-algorithm-based design tool for brushless excitation systems of low-medium rated synchronous generators," Proc. in the $19^{\text {th }}$ International of Electrical Machines and Systems, (ICEMS 2016), 2016

[19] C. Sciascera, P. Giangrande, L. Papini, C. Gerada, and M. Galea, "Analytical Thermal Model for Fast Stator Winding Temperature Prediction," in IEEE Transactions on Industrial Electronics, vol. 64, n. 8, pp. 6116-6126, March 2017.

[20] A. Al-Timimy, P. Giangrande, M. Degano, Z. Xu, M. Galea, C. Gerada, G. Lo Calzo, H. Zheng, and L. Xia, "Design and Losses Analysis of a High Power Density Machine for Flooded Pump Applications" in IEEE Transaction On Industry Applications, vol. 54, no. 4, pp. 3260-3270, 2018.

[21] S.A Odhano, P. Giangrande, R. Bojoi and C. Gerada, "Selfcommissioning of interior permanent magnet synchronous motor drives with high-frequency current injection", 5th Annual IEEE Energy Conversion Congress and Exhibition, Pages 3852-3859, 2013. 